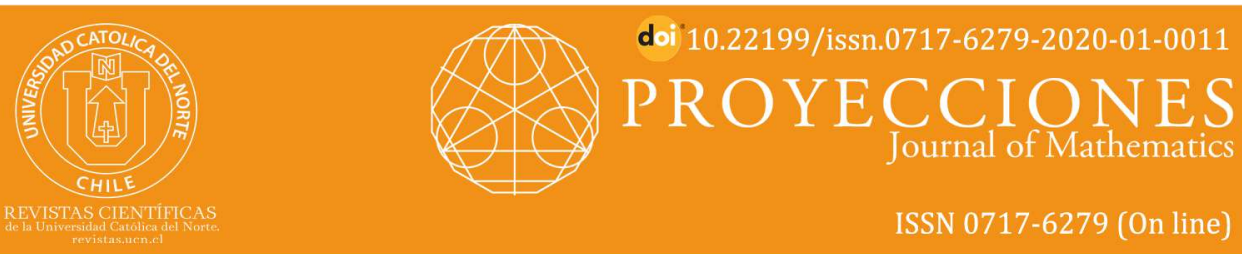

\title{
The total double geodetic number of a graph
}

\section{A. P. Santhakumaran ${ }^{1}$ ๑) orcid.org/0000-0002-8639-1246 T. Jebaraj ${ }^{2}$}

${ }^{1}$ Hindustan Institute of Technology and Science, Dept. of Mathematics, Chennai, TN, India.

apskumar1953@gmail.com

${ }^{2}$ Malankara Catholic College, Dept. of Mathematics, Kaliyakavilai, TN, India.

vjebaraj.math@gmail.com

Received: January2019 | Accepted: August 2019

\section{Abstract:}

For a connected graph $G$ of order n, a set $S$ of vertices is called a double geodetic set of $G$ if for each pair of vertices $x, y$ in $G$ there exist vertices $u, v \in S$ such that $x, y \in I[u, v]$. The double geodetic number $\operatorname{dg}(G)$ is the minimum cardinality of a double geodetic set. Any double godetic set of cardinality $\operatorname{dg}(G)$ is called a dg-set of $G$. A connected double geodetic set of $G$ is a double geodetic set $S$ such that the subgraph G[S] induced by $S$ is connected. The mínimum cardinality of a connected double geodetic set of $G$ is the connected double geodetic number of $G$ and is denoted by $\operatorname{dgc}(G)$. A connected double geodetic set of cardinality $\operatorname{dgc}(G)$ is called a dgc-set of $G$. A total double geodetic set of a graph $G$ is a double geodetic set $S$ such that the subgraph $G[S]$ induced by $S$ has no isolated vertices. The minimum cardinality of a total double geodetic set of $G$ is the total double geodetic number of $G$ and is denoted by $\operatorname{dgt}(G)$. For positive integers $r, d$ and $k \geq 4$ with $r \leq d \leq 2 r$, there exists a connected graph $G$ with $\operatorname{rad} G=r, \operatorname{diam} G=d$ and $\operatorname{dgt}(G)=k$. It is shown that if $n, a, b$ are positive integers such that $4 \leq a \leq b \leq n$, then there exists a connected graph $G$ of order $n$ with $\operatorname{dgt}(G)=a$ and $\operatorname{dgc}(G)=b$. Also, for integers $a, b$ with $4 \leq a \leq b$ and $b \leq 2 a$, there exists a connected graph $G$ such that $\operatorname{dg}(G)=a$ and $\operatorname{dgt}(G)=b$.

Keywords: Geodetic number; Double geodetic number; Connected double geodetic number; Total double geodetic number.

MSC (2010): 05C12.

\section{Cite this article as (IEEE citation style)}

A. P. Santhakumaran and T. Jebaraj, "The total double geo-

detic number of a graph", Proyecciones (Antofagasta, On

line), vol. 39, no. 1, pp. 167-178, Feb. 2020, doi:

10.22199/issn.0717-6279-2020-01-0011. [Accessed ddmm-yyyy].

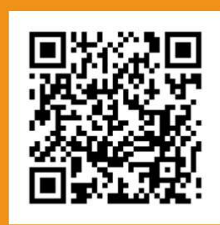

Article copyright: (C) 2020 A. P. Santhakumaran and T. Jebaraj. This is an open access article distributed under the terms of the Creative Commons Licence, which permits unrestricted use and distribution provided the original author and source are credited.

(cc) BY 


\section{Introduction}

By a graph $G=(V, E)$ we mean a finite undirected connected graph without loops or multiple edges. The order and size of $G$ are denoted by $n$ and $m$, respectively. For basic graph theoretic terminology we refer to [4]. For vertices $x$ and $y$ in a connected graph $G$, the distance $d(x, y)$ is the length of a shortest $x-y$ path in $G$. It is known that the distance $d$ is a metric on the vertex set of $G$. An $x-y$ path of length $d(x, y)$ is called an $x-y$ geodesic. A vertex $v$ is said to lie on an $x$-y geodesic $P$ if $v$ is a vertex of $P$ including the vertices $x$ and $y$. For any vertex $u$ of $G$, the eccentricity of $u$ is $e(u)=\max \{d(u, v): v \in V\}$. A vertex $v$ is an eccentric vertex of $u$ if $e(u)=d(u, v)$. The radius $\mathrm{rad} G$ and diameter diam $G$ are defined by $\operatorname{rad} G=\min \{e(v): v \in V\}$ and $\operatorname{diam} G=\max \{e(v): v \in V\}$, respectively. The neighborhood of a vertex $v$ is the set $N(v)$ consisting of all vertices $u$ which are adjacent with $v$. A vertex $v$ is an extreme vertex of $G$ if the subgraph induced $N(v)$ is complete. The closed interval $I[x, y]$ consists of all vertices lying on some $x-y$ geodesic of $G$. A vertex $v$ is a weak extreme vertex of $G$ if there exists a vertex $u$ in $G$ such that $u, v \in I[x, y]$ for a pair of vertices $x, y$ in $G$, then $v=x$ or $v=y$. Equivalently, a vertex $v$ in a connected graph is a weak extreme vertex if there exists a vertex $u$ in $G$ such that $v$ is either an initial vertex or a terminal vertex of any interval containing both $u$ and $v$. Each extreme vertex of a graph is weak extreme. For the graph $G$ in Figure 1, it is easily seen that each vertex is weak extreme. However, no vertex of $G$ is extreme.

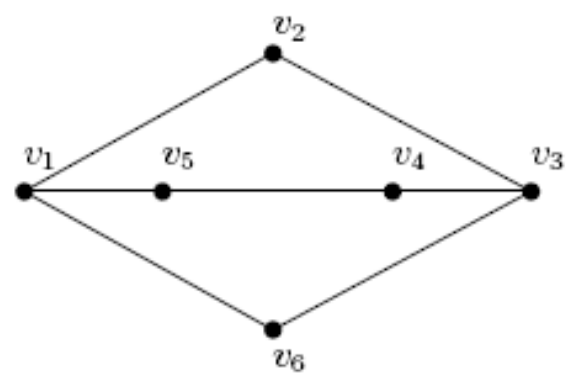

Figure 1: $G$ 
For $S \subseteq V$, let $I[S]=\bigcup_{x, y \in S} I[x, y]$. A set $S$ of vertices is a geodetic set of $G$ if $I[S]=V$, and the minimum cardinality of a geodetic set is the geodetic number $g(G)$. A geodetic set of cardinality $g(G)$ is called a $g$-set of $G$. A total geodetic set $S$ of $G$ is a geodetic set such that the subgraph $G[S]$ induced by $S$ has no isolated vertices. The minimum cardinality of a total geodetic set of $G$ is the total geodetic number of $G$ and is denoted by $g_{t}(G)$. A total geodetic set of cardinality $g_{t}(G)$ is called a $g_{t}$-set of $G$. The geodetic number of a graph was introduced in $[1,5]$ and further studied in $[2,3,6]$. Let $2^{V}$ denote the set of all subsets of $V$. The mapping $I: V \times V \rightarrow$ $2^{V}$ defined by $I[u, v]=\{w \in V:$ w lies on a $u-v$ geodesic in $G\}$ is the interval function of $G$. One of the basic properties of $I$ is that $u, v \in I[u, v]$ for any pair $u, v \in V$. Hence the interval function captures every pair of vertices and so the problem of double geodetic sets is trivially well-defined while it is clear that this fails in many graphs already for triplets (for example, complete graphs). This is the motivation for introducing and studying double geodetic sets.

A set $S$ of vertices in $G$ is called a double geodetic set of $G$ if for each pair of vertices $x, y$ there exist vertices $u, v \in S$ such that $x, y \in I[u, v]$. The double geodetic number $\operatorname{dg}(G)$ is the minimum cardinality of a double geodetic set. Any double geodetic of cardinality $d g(G)$ is called a $d g$-set of $G$. The double geodetic number of a graph was introduced and studied in [8]. The following theorems will be used in the sequel.

Theorem 1.1. [8] Every double geodetic set of a connected graph $G$ contains all the weak extreme vertices of $G$. In particular, if the set $W$ of all weak extreme vertices is a double geodetic set, then $W$ is the unique $d g$-set of $G$.

Theorem 1.2. [8] For the odd cycle $G=C_{2 n+1}(n \geq 1), d g(G)=2 n+1$.

Theorem 1.3. [9] Each cutvertex of a connected graph $G$ belongs to every connected double geodetic set of $G$.

\section{The total double geodetic number of a graph}

Definition 2.1. Let $G$ be a connected graph with at least two vertices. A total double geodetic set of a graph $G$ is a double geodetic set $S$ such that the subgraph $G[S]$ induced by $S$ has no isolated vertices. The minimum cardinality of a total double geodetic set of $G$ is the total double geodetic number of $G$ and is denoted by $d g_{t}(G)$. 
Example 2.2. For the graph $G$ given in Figure 2.1, $S=\left\{v_{1}, v_{2}, v_{7}, v_{6}\right\}$ is the minimum double geodetic set of $G$ so that $d g(G)=4$. Note that the subgraph induced by $S$ has isolated vertices so that $S$ is not a total double geodetic set of $G$. It is easily seen that $T=\left\{v_{1}, v_{2}, v_{3}, v_{7}, v_{5}, v_{6}\right\}$ is a minimum total double geodetic set of $G$ and so $d g_{t}(G)=6$.

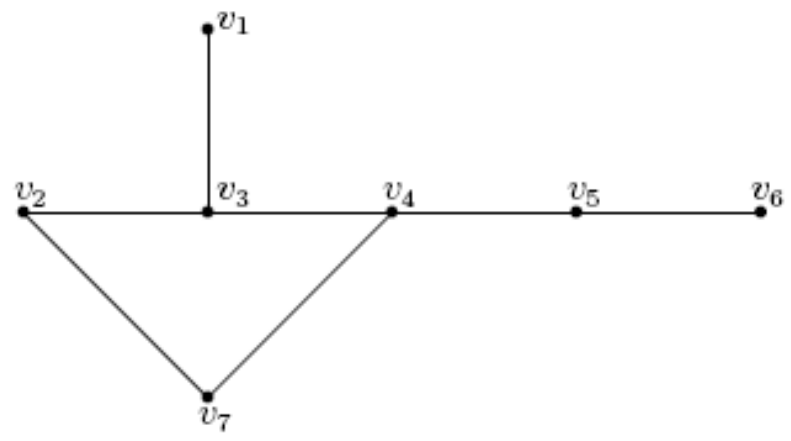

Figure 2.1: $G$

Further, it is easily verified that $U=\left\{v_{1}, v_{2}, v_{3}, v_{4}, v_{5}, v_{6}, v_{7}\right\}$ is the unique minimum connected double geodetic set of $G$ so that $d g_{c}(G)=7$ for the graph $G$ given in Figure 2.1. Thus for the graph $G$ given in Figure 2.1, $d g(G)=4, d g_{t}(G)=6$ and $d g_{c}(G)=7$.

It follows from Theorem 1.1 that every weak extreme vertex of a connected graph $G$ belongs to every total double geodetic set of $G$. Hence for the complete graph $K_{n}(n \geq 2), d g_{t}\left(K_{n}\right)=n$.

Theorem 2.3. For a connected graph $G$ of order $n, 2 \leq d g(G) \leq d g_{t}(G) \leq$ $d g_{c}(G) \leq n$.

Proof. Any double geodetic set needs at least two vertices and so $d g(G) \geq 2$. Since every total double geodetic set is a double geodetic set, we have $d g(G) \leq d g_{t}(G)$, and every connected double geodetic is a total double geodetic set of $G$ so that $d g_{t}(G) \leq d g_{c}(G)$. Also, since $G$ is connected, it is clear that $d g_{c}(G) \leq n$. Thus $2 \leq d g(G) \leq d g_{t}(G) \leq d g_{c}(G) \leq n$.

A vertex $v$ of a connected graph $G$ is called a support of $G$ if it is adjacent to an endvertex of $G$. 
Theorem 2.4. Every total double geodetic set of a connected graph $G$ contains all the weak extreme vertices and the support vertices of $G$. In particular, if the set $S$ of all weak extreme vertices and support vertices is a total double geodtic set, then $S$ is the unique $d g_{t}$-set of $G$.

Proof. This follows from Theorem 1.1.

For a nontrivial tree $T$, the set $S$ of all endvertices and all support vertices forms a total double geodetic set of $T$ so that $d g_{t}(T)=|S|$.

Theorem 2.5. For a nontrivial tree $T$ of order $n, d g_{t}(T)=n$ if and only if $T$ is a caterpillar with all its cutvertices are support vertices.

Proof. Let $d g_{t}(T)=n$. By Theorem 2.4, all the vertices of $T$ are end vertices and support vertices. Hence it follows that $T$ is a caterpillar. The converse part is clear.

Theorem 2.6. For the complete bipartite graph $G=K_{m, n}$,

$\operatorname{dg}_{t}(G)= \begin{cases}2 & \text { if } m=n=1 \\ n+1 & \text { if } m=1, n \geq 2 \\ \min \{m, n\}+1 & \text { if } m, n \geq 2\end{cases}$

Proof. The first two parts are clear as $G$ is a tree. For $m, n \geq 2$, let $X=$ $\left\{x_{1}, x_{2}\right.$,

$\left.\ldots, x_{m}\right\}, Y=\left\{y_{1}, y_{2}, \ldots, y_{n}\right\}$ be the partite sets of $G$. Let $S$ be a double geodetic set of $G$. We claim that $X \subseteq S$ or $Y \subseteq S$. Otherwise, there exist vertices $x, y$ such that $x \in X, y \in Y$ and $x, y \notin S$. Now, since the pair of vertices $x, y$ lie only on the intervals $I[x, y], I[x, t]$ or $I[s, y]$ for some $t \in X$ and $s \in Y$, it follows that $x \in S$ or $y \in S$, which is a contradiction. Hence $X \subseteq S$ or $Y \subseteq S$. Also it is clear that both $X$ and $Y$ are double geodetic sets of $K_{m, n}$ and so $d g(G)=\min \{m, n\}$. Assume that $m \leq n$. Then $S=X \cup\{y\}$, where $y \in Y$, is a total double geodtic set of $G$ and so $d g_{t}(G)=m+1=\min \{m, n\}+1$.

Theorem 2.7. For an even cycle $G=C_{2 n}, \quad d g_{t}(G)= \begin{cases}3 & \text { if } n=2 \\ 4 & \text { if } n \geq 3\end{cases}$ 
Proof. For $n=2$, it is clear that any set of three vertices is a minimum total double geodetic set of $G$ so that $d g_{t}(G)=3$. For $n \geq 3$, let $G$ be the cycle $C_{2 n}: v_{1}, v_{2}, \ldots, v_{n}, v_{n+1} \ldots, v_{2 n}, v_{1}$. Since $S=\left\{v_{1}, v_{n+1}\right\}$ is a double geodetic set, it is easily seen that $S_{1}=S \cup\left\{v_{2}, v_{n}\right\}$ is a minimum total double geodetic set of $G$ so that $d g_{t}(G)=4$.

Theorem 2.8. For the odd cycle $G=C_{2 n+1}(n \geq 1), d g_{t}(G)=2 n+1$.

Proof. This follows from Theorem 1.2.

Theorem 2.9. For any connected graph $G, d g_{t}(G)=2$ if and only if $G=K_{2}$.

Proof. Let $d g_{t}(G)=2$ and let $S=\{u, v\}$ be a total double geodetic set of $G$. Then $u v$ is an edge. It is clear that a vertex different from $u$ and $v$ cannot lie on a $u-v$ geodesic and so $G=K_{2}$. The converse is clear.

Theorem 2.10. Let $G$ be a connected graph with at least two vertices. Then $d g_{t}(G) \leq 2 d g(G)$.

Proof. Let $S=\left\{v_{1}, v_{2}, \ldots, v_{k}\right\}$ be a minimum double geodetic set of $G$. Let $u_{i} \in N\left(v_{i}\right)$ for $i=1,2, \ldots, k$ and $T=\left\{u_{1}, u_{2}, \ldots, u_{k}\right\}$. Then $S \cup T$ is a total double geodtic set of $G$ so that $d g_{t}(G) \leq|S \cup T| \leq 2 k=2 d g(G)$.

In view of Theorem 2.10, we have the following realization result.

Theorem 2.11. For integers $a, b$ with $4 \leq a \leq b$ and $b \leq 2 a$, there exists a connected graph $G$ such that $d g(G)=a$ and $d g_{t}(G)=b$.

Proof. Case 1. For $a=b$, the complete graph $K_{a}$ has the desired properties.

Case 2. $a<b$. Let $b=a+p$, where $1 \leq p \leq a$. For $p=1$, the star $K_{1, a}$ has the desired properties. Now, let $p \geq 2$. For each integer $i$ with $1 \leq i \leq p-1$, let $F_{i}$ be a copy of the cycle $C_{4}$ with vertex set $V\left(F_{i}\right)=\left\{x_{i}, y_{i}, z_{i}, w_{i}\right\}$. Let $H$ be a graph formed by identifying all these $F_{i}$ at $x_{i}$, and let $x$ be the identified vertices. Let $P_{b}: u_{1}, u_{2}, \ldots, u_{b}$ be a path of order $b$. Let $H^{\prime}$ be the graph obtained by identifying the vertex $u_{1}$ of $P_{b}$ with the vertex $x$ of $H$. Let $G$ be the graph obtained from $H^{\prime}$ by adding the new vertices $v_{1}, v_{2}, \ldots, v_{a-p}$, and joining $v_{1}$ with $u_{b-1}$, and each $v_{i}(2 \leq i \leq a-p)$ with both $u_{b-2}$ and $u_{b}$. The graph $G$ is shown in 
Figure 2.2. Let $S=\left\{v_{1}, v_{2}, \ldots, v_{a-p}, u_{b}, z_{1}, z_{2}, \ldots, z_{p-1}\right\}$ be the set of all weak extreme vertices of $G$. Since $S$ is a double geodetic set of $G$, it follows from Theorem 1.1 that $d g(G)=a$.

By Theorem 2.4, every total double geodetic set of $G$ contains $S$. It is clear that $S_{1}=S \cup\left\{y_{1}, y_{2}, \ldots, y_{p-1}, u_{b-1}\right\}$ is a minimum total double geodetic set of $G$ so that $d g_{t}(G)=b$.

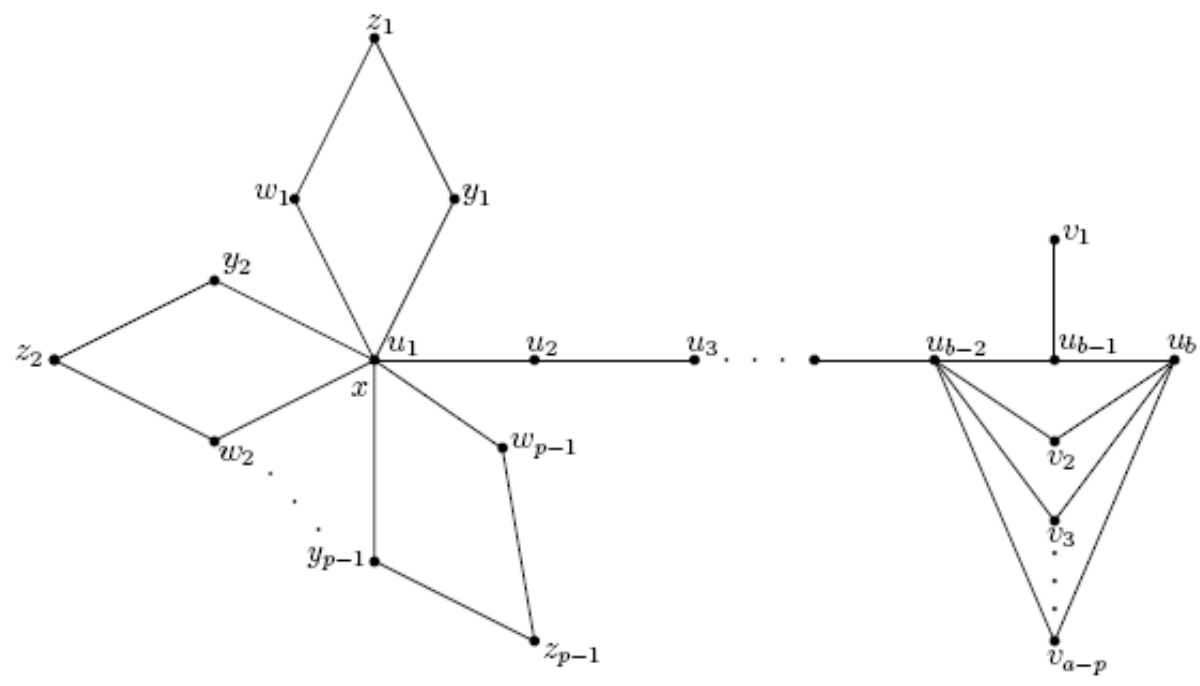

Figure 2.2: $G$

For every connected graph $G, \operatorname{rad} G \leq \operatorname{diam} G \leq 2 \mathrm{rad} G$. Ostrand [7] showed that every two positive integers $a$ and $b$ with $a \leq b \leq 2 a$ are realizable as the radius and diameter, respectively of some connected graph. Now, Ostrand's theorem can be extended so that the total double geodetic number can also be prescribed.

Theorem 2.12. For positive integers $r, d$ and $k \geq 4$ with $r \leq d \leq 2 r$, there exists a connected graph $G$ with $\operatorname{rad} G=r$, diam $G=d$ and $d g_{t}(G)=k$.

Proof. If $r=1$, then $d=1$ or 2. For $d=1$, let $G=K_{k}$. Then $d g_{t}(G)=k$. For $d=2$, let $G=K_{1, k-1}$. Then $d g_{t}(G)=k$. Now, let $r \geq 2$. We construct a graph $G$ with the desired properties as follows.

Case 1. $r=d$. Let $C_{2 r}: u_{1}, u_{2}, \ldots, u_{2 r}, u_{1}$ be a cycle of order $2 r$. Let $G$ be the graph given in Figure 2.3, obtained by adding the new vertices $v_{1}, v_{2}, \ldots, v_{k-3}$ and joining each $v_{i}(1 \leq i \leq k-3)$ with $u_{1}$ and $u_{2 r}$ of $C_{2 r}$. 
It is easily verfied that the eccentricity of each vertex of $G$ is $r$ so that $\operatorname{rad} G=\operatorname{diam} G=r$. Let $S=\left\{v_{1}, v_{2}, \ldots, v_{k-3}, u_{r+1}\right\}$ be the set of all weak extreme vertices of $G$. By Theorem 1.1, every total double geodetic set of $G$ contains $S$. It is clear that for any $x \notin S, S \cup\{x\}$ is not a total double geodetic set of $G$. Since $S_{1}=S \cup\left\{u_{1}, u_{r}\right\}$ is a total double geodetic set of $G$, we have, $d g_{t}(G)=k$.

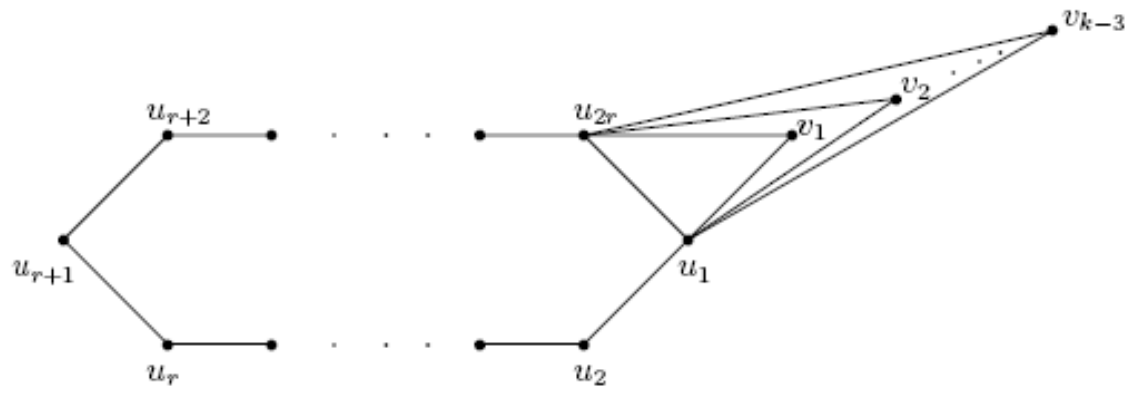

Figure 2.3: $G$

Case 2. $r<d$. First, assume that $k \geq 7$. Let $C_{2 r}: u_{1}, u_{2}, \ldots, u_{2 r}, u_{1}$ be a cycle of order $2 r$ and let $P_{d-r+1}: v_{0}, v_{1}, \ldots, v_{d-r}$ be a path of order $d-r+1$. Let $H$ be the graph obtained from $C_{2 r}$ and $P_{d-r+1}$ by identifying $v_{0}$ of $P_{d-r+1}$ and $u_{1}$ of $C_{2 r}$. Now, add $k-6$ new vertices $w_{1}, w_{2}, \ldots, w_{k-6}$ to the graph $H$ and join $w_{1}$ to $u_{r}$, and join each vertex $w_{i}(2 \leq i \leq k-6)$ to both $u_{r+1}$ and $u_{r-1}$, thereby obtaining the graph $G$ in Figure 2.4. Then $\operatorname{rad} G=$ $r$ and $\operatorname{diam} G=d$. Let $S_{1}=\left\{w_{1}, w_{2}, \ldots, w_{k-6}, u_{r+1}, u_{2 r}, v_{d-r}, u_{r}, v_{d-r-1}\right\}$ be set of all weak extreme vertices and support vertices of $G$. By Theorem 2.4 , every total double geodetic set contains $S_{1}$. It is clear that $S_{1}$ is not a total double geodetic set of $G$. Since $S_{2}=S_{1} \cup\left\{u_{1}\right\}$ is a total double geodetic set of $G$, we have $d g_{t}(G)=k$. 


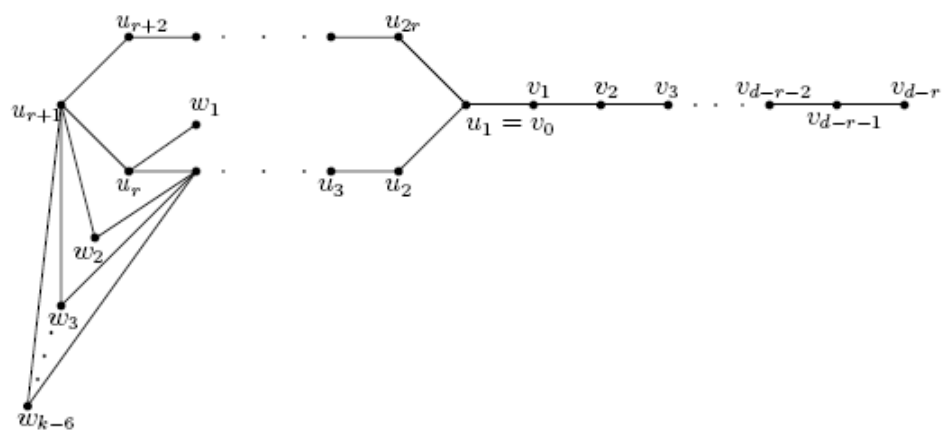

Figure 2.4: $G$

Now, for $k=4,5,6$, let $G_{1}$ be the graph obtained from $H$ by adding $(k-4)$ new vertices $y_{1}, y_{2}, \ldots, y_{k-4}$ and joining each $y_{i}(1 \leq i \leq k-4)$ to both $u_{r}$ and $u_{r+2}$. Then $\operatorname{rad} G_{1}=r$ and diam $G_{1}=d$. Let $T=$ $\left\{y_{1}, y_{2}, \ldots, y_{k-4}, u_{r+1}, v_{d-r-1}, v_{d-r}\right\}$ be the set of all weak extreme vertices and support vertices of $G_{1}$. By Theorem 2.4, every total double geodetic set contains $T$. It is clear that $T_{1}=T \cup\left\{u_{r}\right\}$ is a minimum total double geodetic set of $G_{1}$ so that $d g_{t}(G)=k$.

Theorem 2.13. If $n, a, b$ are integers such that $4 \leq a \leq b \leq n$, then there exists a connected graph $G$ of order $n$ with $d g_{t}(G)=a$ and $d g_{c}(G)=b$.

Proof. We prove this theorem by considering four cases.

Case 1. $a=b=n$. Let $G=K_{n}$. Then $d g_{t}(G)=d g_{c}(G)=n$.

Case 2. $a<b<n$. Let $P_{b-a+4}: u_{1}, u_{2}, \ldots, u_{b-a+4}$ be a path of order $b-a+4$. Add $n+a-b-4$ new vertices $v_{1}, v_{2}, \ldots, v_{n-b}, w_{1}, w_{2}, \ldots, w_{a-4}$ to $P_{b-a+4}$ and join $w_{1}$ to $u_{2}$, and join $w_{2}, w_{3}, \ldots, w_{a-4}$ with $u_{1}$ and $u_{3}$ and also join $v_{1}, v_{2}, \ldots, v_{n-b}$ with both $u_{2}$ and $u_{4}$ to get the graph $G$ of order $n$ given in Figure 2.5. Let $S=\left\{w_{1}, w_{2}, \ldots, w_{a-4}, u_{1}, u_{2}, u_{b-a+3}, u_{b-a+4}\right\}$ be the set of all weak extreme vertices and support vertices of $G$. Since $S$ is a total double geodetic set of $G$, by Theorem $2.4, d g_{t}(G)=a$. Let $S_{1}=\left\{w_{1}, w_{2}, \ldots, w_{a-4}, u_{1}, u_{b-a+4}, u_{2}, u_{4}, u_{5}, \ldots, u_{b-a+3}\right\}$ be the set of all weak extreme vertices and cutvertices. By Theorems 1.1 and 1.3, every connected double geodetic set of $G$ contains $S_{1}$. It is clear $S_{1}$ is not a 
connected double geodetic set of $G$. Since $T=S_{1} \cup\left\{u_{3}\right\}$ is a minimum connected double geodetic set of $G$, we have $d g_{c}(G)=b$.

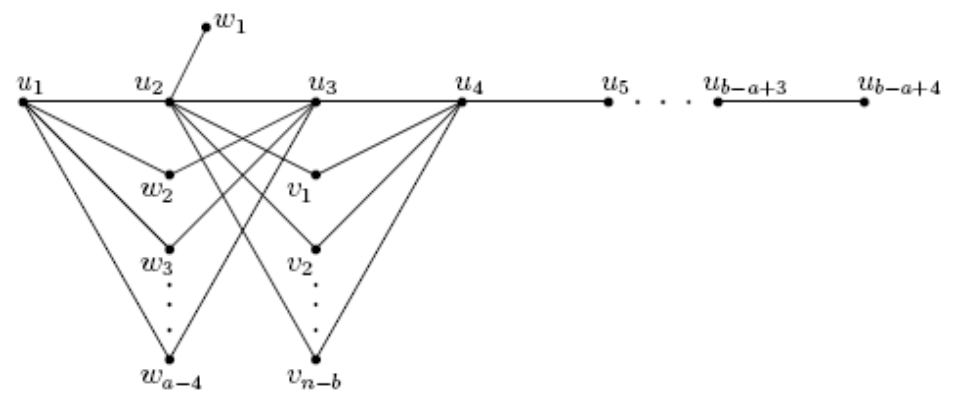

Figure 2.5: $G$

Case 3. $a=b<n$. Let $P_{3}: u_{1}, u_{2}, u_{3}$ be a path of order 3. Add the new vertices $v_{1}, v_{2}, \ldots, v_{n-a}$ and join each $v_{i}(1 \leq i \leq n-a)$ with $u_{1}$ and $u_{3}$. Also, add new vertices $w_{1}, w_{2}, \ldots, w_{a-3}$ and join each $w_{i}(1 \leq i \leq a-3)$ with $u_{1}$, thereby obtaining the graph $G$ of order $n$ given in Figure 2.6.

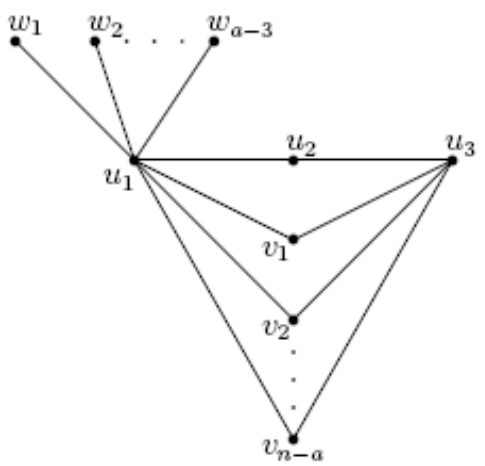

Figure 2.6: $G$ 
Let $S_{2}=\left\{w_{1}, w_{2}, \ldots, w_{a-3}, u_{1}, u_{3}\right\}$ be the set of all weak extreme vertices and support vertices of $G$. By Theorems 1.3 and 2.4, every total double geodetic set as well as every connected double geodetic set of $G$ contains $S_{2}$. Since $S_{2}$ is neither a total double geodetic set nor a connected double geodetic set of $G$ and since $S_{2} \cup\left\{u_{2}\right\}$ is a connected double geodetic set of $G$, we have $d g_{c}(G)=d g_{t}(G)=a=b$.

Case 4. $a<b=n$. Let $P_{b-a+4}: u_{1}, u_{2}, \ldots, u_{b-a+4}$ be a path of order $b-a+4$. Add $a-4$ new vertices $v_{1}, v_{2}, \ldots, v_{a-4}$ to $P_{b-a+4}$ and join $v_{1}$ to $u_{b-a+3}$ and join $v_{2}, v_{3}, \ldots, v_{a-4}$ with both $u_{b-a+2}$ and $u_{b-a+4}$, thereby producing the graph $G$ of order $n$ given in Figure 2.7. Let $S_{3}=$ $\left\{u_{1}, u_{2}, v_{1}, v_{2}, \ldots, v_{a-4}, u_{b-a+3}, u_{b-a+4}\right\}$ be the set of all weak extreme vertices and support vertices of $G$. Since $S_{3}$ is a total double geodetic set of $G$, it follows from Theorem 2.4 that $d g_{t}(G)=a$. Since each vertex of $G$ is either a weak extreme vertex or a cutvertex of $G$, it follows from Theorems 1.1 and 1.3 that $d g_{c}(G)=n=$

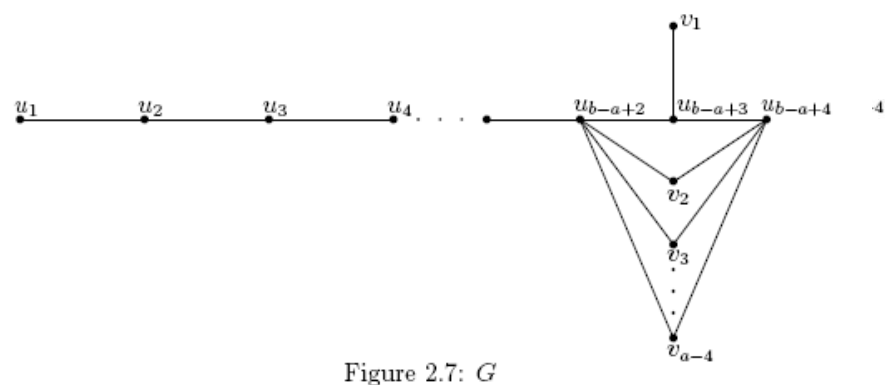

\section{Acknowledgement}

The authors are thankful to the reviewer for the useful comments for the improvement of this paper.

\section{References}

[1] F. Buckley and F. Harary, Distance in graphs, Redwood City, CA: Addison-Wesley, 1990.

[2] G. Chartrand, F. Harary and P. Zhang, "On the geodetic number of a graph", Networks, vol. 39, no. 1, pp. 1-6, Nov. 2002, doi: $10.1002 /$ net.10007. 
[3] G. Chartrand, F. Harary , H. C. Swart and P. Zhang, "Geodomination in graphs", Bulletin of the ICA, vol. 31, pp. 51-59, 2001.

[4] F. Harary, Graph theory, Reading, MA: Addision-Wesley, 1969.

[5] F. Harary, E. Loukakis, and C. Tsouros, "The geodetic number of a graph", Mathematical and computer modeling, vol. 17, no. 11, pp. 89-95, Jun. 1993, doi: 10.1016/0895-7177(93)90259-2.

[6] R. Muntean and P. Zhang, "On geodomonation in graphs", Congressus numerantium, vol. 143, pp. 161-174, 2000.

[7] P. A. Ostrand, "Graphs with specified radius and diameter", Discrete mathematics, vol. 4, no. 1 , pp. $71-75$, 1973, doi: 10.1016/0012365X(73)90116-7.

[8] A. P. Santhakumaran and T. Jebaraj, "Double geodetic number of a graph", Discussiones mathematicae graph theory, vol. 32, no. 1, pp. 109119,2012 . [On line]. Available: https://bit.ly/20cj0uW

[9] A. P. Santhakumaran and T. Jebaraj, "The connected double geodetic number of a graph", (communicated). 